\title{
The problem of credibility studies on deformations of engineering objects in the geologically unstable area of Szczecin
}

\author{
Marek Zygmunt ${ }^{1, *}$, Stefan Cacon $^{1}$, Andrzej Piotrowski ${ }^{2}$ and Grzegorz Stępień ${ }^{1}$ \\ ${ }^{1}$ Maritime University of Szczecin, Institute of Geoinformatics, 46 Żołnierska St., 71-250 Szczecin, Poland \\ ${ }^{2}$ Polish Geological Institute - National Research Institute, Pomeranian Branch, 20 Wieniawskiego St., 71-130 Szczecin, Poland
}

\begin{abstract}
The location of reference points in deformation studies of engineering objects is often associated with low reliability of the obtained measurement results. This concerns the lack of proper diagnosis of the geological structure of the area. The reliability of deformation measurements is also low when we obtain data that only characterize the effects and not the cause-and-effects. The authors reviewed the influence of geological conditions on the formation of deformations of some engineering objects. The reference points were located in the immediate vicinity of the facilities, without taking into account the geological structure of the areas where the facilities were located. The proposed test method is based on a three-segment control and measurement system. An example of such considerations is the engineering facilities on the Grodzka and Ostrów Grabowski Island in the Szczecin area. The basic issue is to locate geologically stable areas in the vicinity of monitored engineering objects on the basis of geological substrate assessment and to analyse archival materials concerning periodical measurements of class 1 and 2 levelling lines in the Szczecin area. Reference points are located, which constitute the first segment of the control and measurement system. Subsequent segments of the system are organized with reference to the points of the first segment. This method provides reliable data on deformations of engineering objects.
\end{abstract}

\section{Introduction}

Local control measurements of major buildings carried out in the cities are usually related to the closest points of the surveying grid [1]. Geodynamic processes can cause surface deformations in areas that significantly exceed local measurements.

Accurate recognition of the geological structure of the area is essential for reliable observation of deformations of engineering objects. A detailed review of the literature concerning the monitoring of object deformations revealed a lack of reference of measurement points to the geological structure of the existing object. Location of measurement points in geologically unstable terrain leads to obtaining data of low reliability. There are no points of stability in the long term. This makes it impossible to interpret the deformation process correctly, as the deformation range cannot be determined. Moreover, it is not possible to identify the cause. This raises the issue of the location and stability of the research network points and the stability of the reference system [2]. Among the time factors, the starting point for deformation measurements is important. It gives the possibility to trace the changes taking place in the intact ground medium. Only then is it possible to interpret the quantitative data like cause and effect but not only effect.

\section{Geodetic monitoring of objects not taking into account their geological structure}

In 1967 there was a catastrophe of a building piling up on the Iwina reservoir in Wroclaw District. 18 people died, 570 of whom were injured. It turned out that a tertiary fault [3], which had not been known to exist during the construction of the facility, occurred under a bursting hole in the reservoir. As a result of the pressure of the copper flotation tailings reservoir on the geologically unstable substrate, seismic shock and fault activation activated. The reference points were located in the immediate vicinity of the tank (Fig. 1), which made it impossible to carry out reliable observations of deformations.

In a similar way, the Nysa Dam was damaged. In 1971 it was put into operation. At the planning and construction stage of the dam, a bundle of tertiary faults activated under the right abutment of the dam was not taken into account. In accordance with the applicable instructions, observations of vertical movements at the dam (commenced in the same year) were carried out by

\footnotetext{
* Corresponding author: marek.zygmunt@op.pl
} 
Meteorology Institute, Wrocław Branch, with respect to the benchmarks located in the foreground of the dam (Fig. 2).

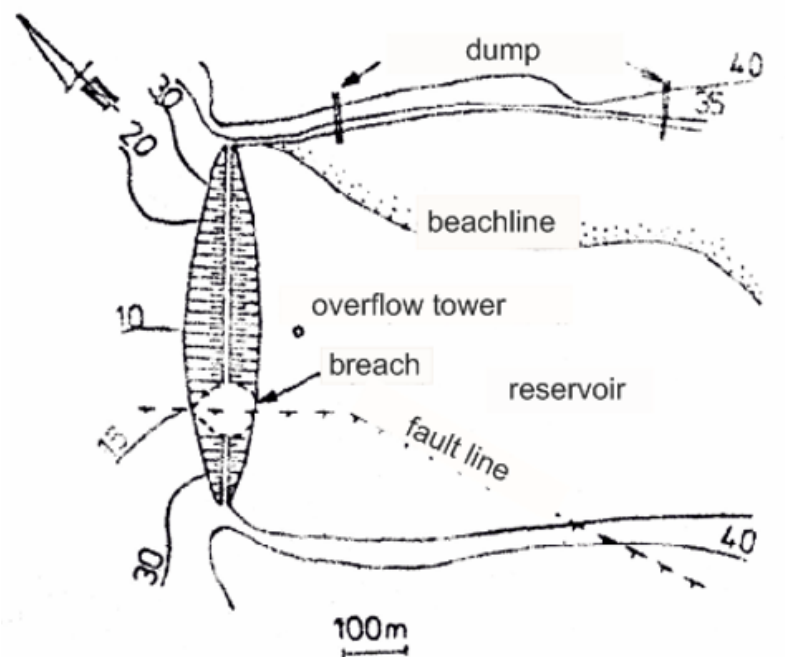

Fig. 1. The interrupted dam of the Konrad Mine near Iwiny [3].

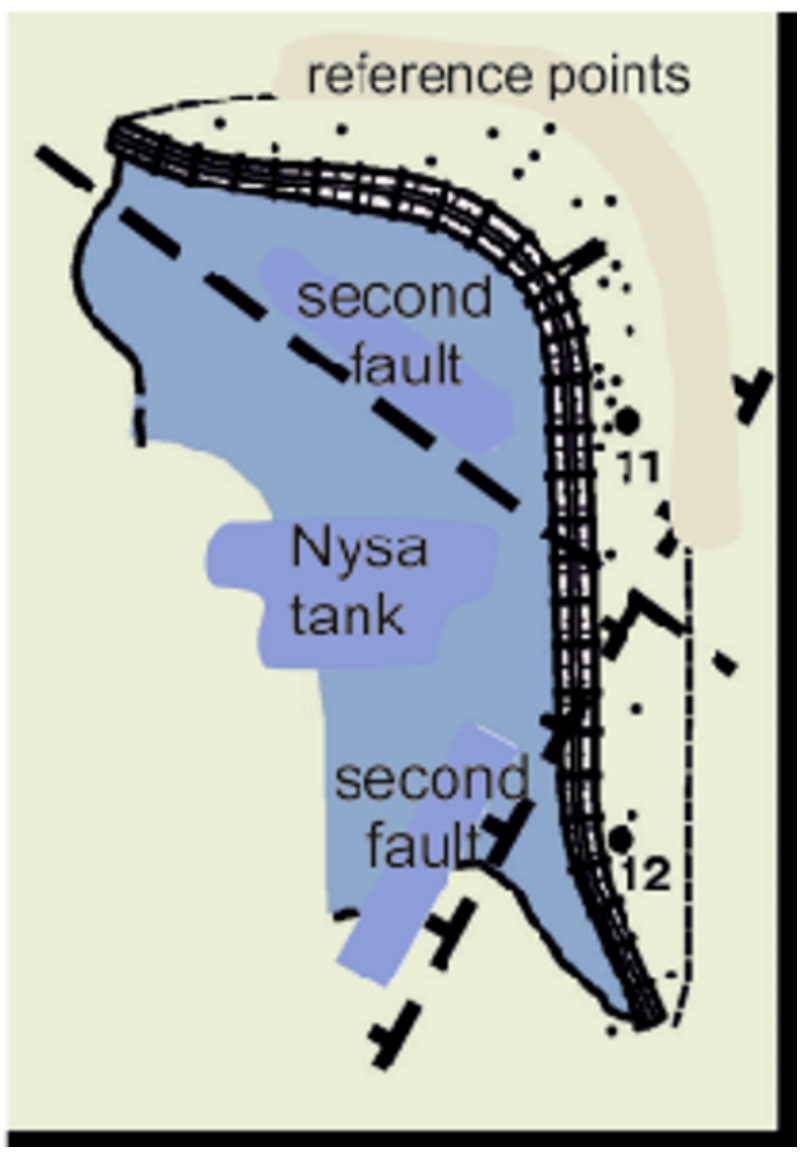

Fig. 2. Location of reference and control points $(11,12)$ on the Nysa Dam [2].

Team S. Cacoń and S. Dyjor [4] revealed irregularities in the organisation of these observations. They were found on the basis of the analysis of archival materials concerning the results of repeated measurements (years 1926, 1953, 1975, 1994) during precise levelling class 1 of Paczków-Nysa, which passed by the analyzed dam. After the Nysa reservoir was put into operation, benchmarks were anomaly deposited below the dam, reaching $-92 \mathrm{~mm}$ in the period 19531975 [2]. This threat was not detected on the basis of standard, periodic measurements of the dam, which did not take into account the geological structure of the object.

\section{Geological deformations in Szczecin}

Examples of deformations of engineering structures built on the geologically unstable Odra Islands in Szczecin are: a damaged bridgehead connecting Grodzka Island with Lasztownia Island and deformations of the handling infrastructure of the municipal waste sorting plant on Zaleskie Łęgi Island (Fig. 3). These deformations were caused by the loading of organic soils (peats, silts, gyttias) by objects placed on them. The water was shrunk and discharged through organic soils [5].
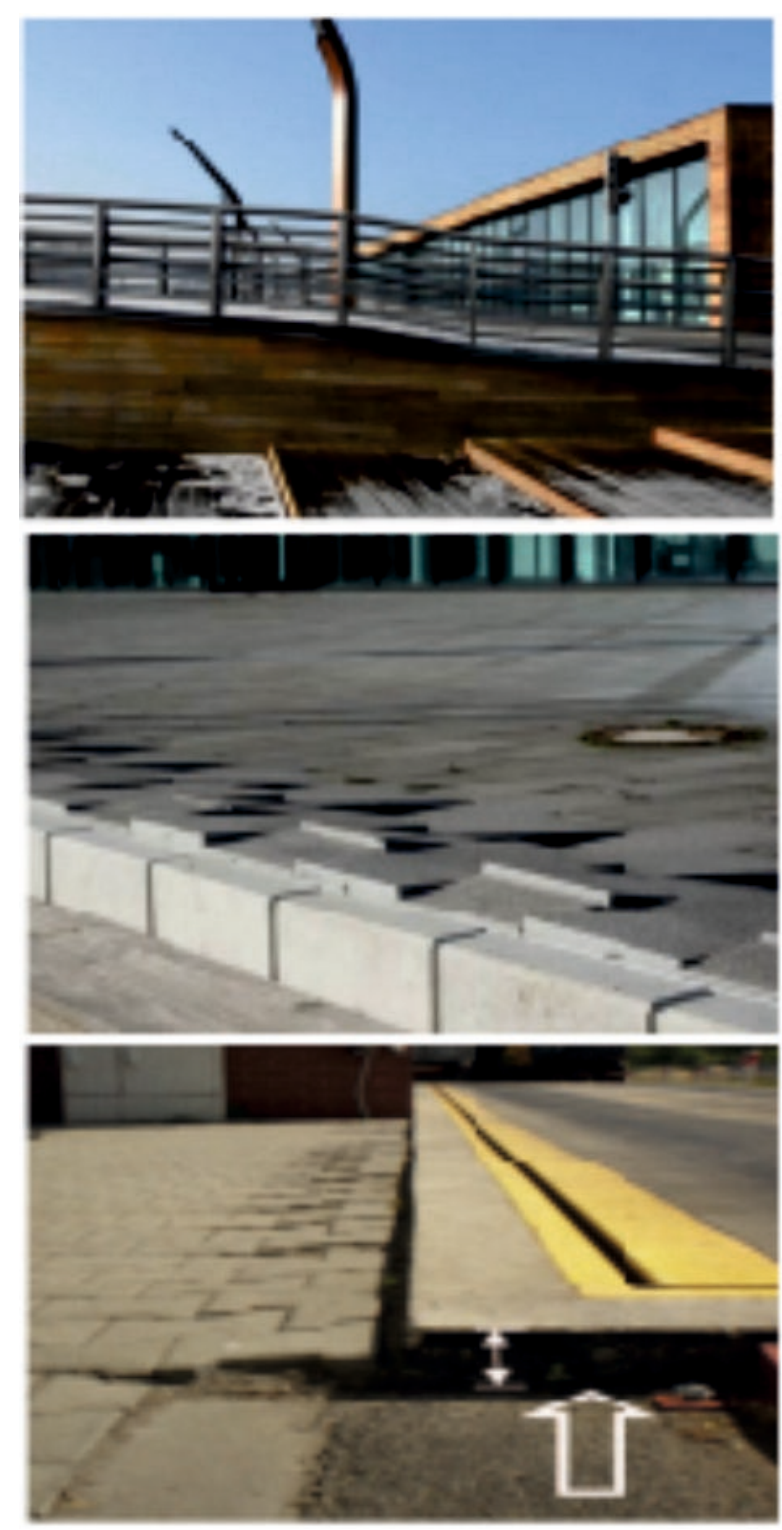

Fig. 3. Examples of object deformations observed in the Szczecin islands $[5,6]$. 
In the case of these objects, no monitoring integrating geodetic observations with the geological structure of the area was carried out, which resulted in deformations visible above.

\section{Factors affecting the reliability of deformation measurements}

The essence of deformation measurements is related to space-time. The factors representing space are the location of the test points, the stability of the test points, the stability of the reference system and the accuracy of the periodic observations. The time factors are: the starting point of the measurements, the time interval between the measurements, the time of the elaborate of the measurements and the time of the preparation of the results [7]. The last two time factors can be reduced due to the use of modern measuring instruments and computers. The location of research network points, their stabilisation, the start of measurements and the time interval are often secondary importance for the surveyor. This is a justified approach if specialists from other fields (geologists, hydrotechnicians) are actively involved in solving such problems [2]. The reliability of deformation measurements in quantitative terms was assessed on the basis of reliability theory [8]. The individual factors $\left(\delta_{i j}\right)$ are considered to be random events and the probability of meeting the reliability conditions is a probabilistic characteristic of the system.

Reliability $W_{i}$ of each system factor is expressed by the probability $P$ of the $\delta_{i}$ not exceeding the $\delta_{i g r}$ limit value:

$$
W_{i}=P\left|\delta_{i} \leqslant \delta_{i g r}\right|=\int_{0}^{i g r} \varphi_{i} \cdot\left(\delta_{i}\right) d \cdot \delta_{i},
$$

where:

$\varphi_{i}\left(\delta_{i}\right)$ - variable probability density function $\delta_{i}$.

When the reliability of one factor drops to the level of 0.67 , then with statistical certainty $\left(W_{i}=0.99\right)$ of the remaining factors the reliability of the whole "system" is low. On the other hand, if the number of analyzed factors is higher or lower, the values of the meters do not change [2].

The reliability of the $\delta_{i}$ factors can be assesed using $t_{\alpha}$ limits of $3,2,1$ :

$$
\begin{gathered}
W_{i(3)}=\left[P\left|\delta_{i} \leq 3 \sigma \delta_{i}\right|=0.99\right] \\
W_{i(2)}=\left[P\left|\delta_{i} \leq 2 \sigma \delta_{i}\right|=0.95\right] \\
W_{i(I)}=\left[P\left|\delta_{i} \leq \sigma \delta_{i}\right|=0.67\right]
\end{gathered}
$$

where:

$W_{i(3)}$ - reliability for the $\delta_{i}$ variable in the range of 3 standard deviations $(3 \sigma)$,

$W_{i(2)}$ - reliability for the $\delta_{i}$ variable in the range of 2 standard deviations $(2 \sigma)$,

$W_{i(1)}$ - reliability for the $\delta_{i}$ variable within 1 standard deviation $(\sigma)$.
The total reliability of the $W_{c}$ of the analyzed measurement is determined by the equation:

$$
W_{c}=\prod_{i=1}^{n} W_{i} \text {, }
$$

where:

$n$ - number of elements of the system.

Determining the probability $\alpha$ of and not exceeding the limit values is related to determining the safety requirements of the facility. This implies taking the function of the probability density $\varphi_{i}$ for individual random variables of $\delta_{i}$. The total reliability of $W_{c}$ can be expressed by the formula:

$$
W_{c}=\prod_{i=1}^{n}\left[P \cdot\left|\delta_{i} \leqslant t_{\alpha} \cdot \sigma \cdot \delta_{i}\right|=\alpha\right]
$$

The following measures of reliability of measurement of the process of deformation of engineering objects have been proposed: high for $W_{c} \geq$ 0.85 , medium for $0.85 \geq W_{c} \geq 0.70$, low for $W_{c} \leq 0.70$.

\section{Proposed solution}

In order to identify the stable points of the first segment of the control and measurement system, a detailed analysis of the geological structure of the city of Szczecin was made (Fig. 4). This made it possible to predefine two geologically stable regions in the northwest and south-eastern parts of the city. Pleistocene and Holocene formations are dominant in the geological structure of the city and its surroundings. The thickness of these formations ranges from $25-30 \mathrm{~m}$ in the area of Niebuszewska Trough and the northern base of the Bukowe Mountains to nearly $200 \mathrm{~m}$ in the area of Warszewskie Hills. Pleistocene settlements are represented by the South-Polish Glaciation, the CentralPolish Glaciation and the North-Polish Glaciation. South-Polish glaciation includes till and Central-Polish glaciation includes clays, sand and mud [9]. Sands and sands with gravel as well as clays on sands are deposits of the northern Poland glaciation. The Holocene is composed of colloquial sediments, diluvial sands and clays, as well as sands and river silt. The youngest sediments are peat silts in drainage basins, as well as sands and lake silt. In the area of the interval period, as a result of the pressure of existing objects on the ground, the layers of organic land were comprimated from 8 to 2 $\mathrm{m}[10,11]$. Older substrate compositions (jura, oligocene) may indirectly affect vertical movements in the city area. As a result of the plastic movements of salts within these compositions, their pressure on surface compositions increases [11]. This can lead to local lifting movements.

Geologically stable areas are located within dense glacial sands and river sands (Fig. 4).

Information on changes in vertical displacement velocity was obtained by analyzing the differences in the height and height of benchmarks from two measurement campaigns of class 1 and 2 levelling lines carried out 
over a period of 25 years. This information was analysed in close connection with the geological structure of the city area. On this basis, 2 stable points were selected, which will provide support for locating reference points of the second segment of the control and measurement system. Changes in the height of benchmarks in the period between measurement campaigns are the basis for calculating the velocity of movement of benchmarks. Points 1-5 (Fig. 5) show the highest (up to 0.52 $\mathrm{mm} /$ year) vertical movement velocities in the southern parts of the islands. In the area of the Oder islands, the areas of existing and planned development have been marked. Deformations of these objects will be recorded by the devices of the second segment of the control and measurement system with reference to geologically stable points of the first segment (Fig. 5).

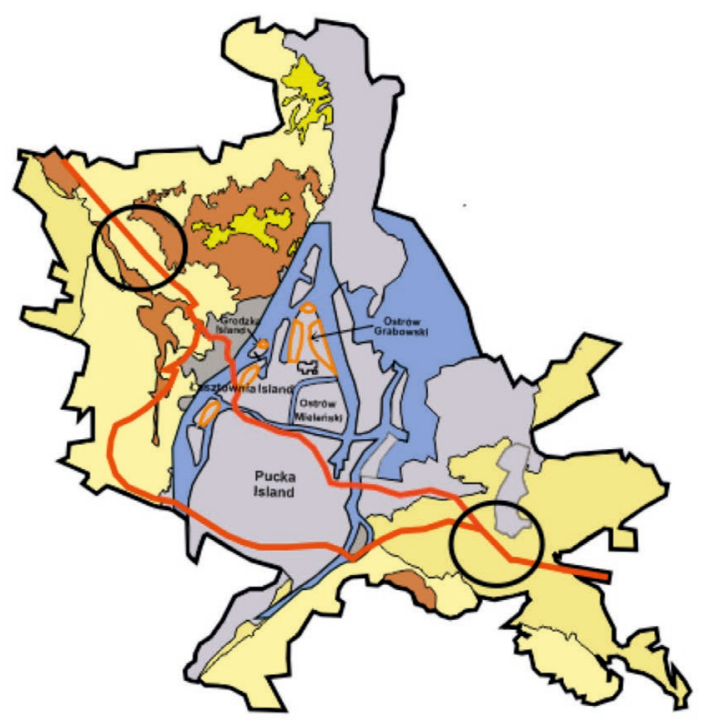

GLACIAL AND HYDRO-GLACIAL SANDS AND GRAVEL
CLAY
LOAMS
ORGANIC LAND (PEATS, SILTS)
SANDS AND GRAVLES
RIVER SANDS
CULTURAL EMBANKMENTS
PLANNED DEVELOPMENTS WITHIN THE ISLANDS
GEOLOGICALLY STABLE AREAS

Fig. 4. Location of geologically stable areas (based on Detailed geological map of Poland, Szczecin sheet).

The following system of monitoring displacements of engineering objects in the space of 1D (Tab. 1) was proposed.

By selecting more stable points in the future, it will be possible to investigate deformations of engineering objects in the space of 3D (vertical and horizontal movements). This will be possible thanks to the use of GNSS-GPS satellite measurements [7].

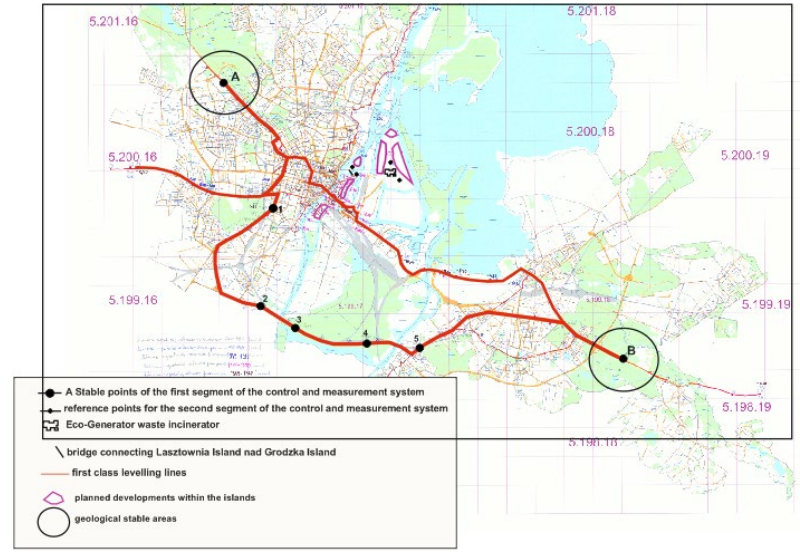

Fig. 5. Stable points constituting the first segment of the control and measurement system.

Table 1. Diagram of the control and measurement system for testing the deformations of $1 \mathrm{D}$ objects.

\begin{tabular}{|c|c|c|c|}
\hline & Segment I & Segment II & Segment III \\
\hline Observations & $\begin{array}{l}\text { Precision } \\
\text { levelling }\end{array}$ & $\begin{array}{c}\text { Total } \\
\text { Station } \\
\text { Precision } \\
\text { levelling }\end{array}$ & $\begin{array}{l}\text { Extensometer } \\
\text { Feeler } \\
\text { (devices of } \\
\text { control and } \\
\text { measurement } \\
\text { system } \\
\text { intended for } \\
\text { objects in } \\
\text { use) }\end{array}$ \\
\hline $\begin{array}{c}\text { Frequency of } \\
\text { observation }\end{array}$ & 1 year & 3 months & $1-30$ days \\
\hline $\begin{array}{c}\text { Accuracy of } \\
\text { displacement } \\
\text { determination }\end{array}$ & $\pm(0.5) \mathrm{mm}$ & $\begin{array}{c} \pm(0.5-2) \\
\mathrm{mm}\end{array}$ & $\begin{array}{c} \pm(0.01-0.1) \\
\mathrm{mm}\end{array}$ \\
\hline
\end{tabular}

\section{Proposed solution}

The stability of the reference system in the measurement of deformations of engineering objects is a prerequisite for obtaining results with the highest reliability.

In the organization of deformation measurements on engineering objects in Szczecin, epochal measurements of class 1 and 2 levelling lines and geological crosssections under these lines were used to locate reference points.

It is important to start monitoring engineering structures already at the stage of investment design. This will enable proper organization of measurements of deformations of the future object and will allow to take into account all spatial and time factors decisive for reliable obtaining of their results. It will be possible to interpret the data in a causal and not only a causal way.

Two stability points shall be provided for testing the deformations of the engineers' structures in a space of 1D (vertical motions). As a result, it is possible to observe the settlement of objects located on the geologically unstable islands of the Oder River. 


\section{References}

1. T. Lazzarini, Geodetic measurements of building displacements and their surroundings (PPWK, Warszawa, 1997, in Polish)

2. S. Cacon, Scientific Works of the Institute of Geodesy and Cartography, vol. XLVIII, issue 102 (2001, in Polish)

3. K. Fidler (Ed.), Failures and catastrophes of dams threats, their causes and effects as well as preventive actions (IMGW Warszawa, 2007, in Polish)

4. S. Cacoń and S. Dyjor, Scientific Papers of the Wrocław University of Agriculture, Geodesy and Agricultural Appliances, XI, 231, 19-31 (1993, in Polish)

5. Z. Meyer, Geotechnical expertise of the bridge connecting Łasztownia Island with the Grodzka Island (2017, in Polish)

6. T. Paczkowska, Building Review, 84, 10, 50-53 (2013)

7. S. Cacoń, Proceedings of XXI International Congress FIG, Commission 6, Brighton UK, 1998, 530-540 (1998)

8. J. Migdalski (Ed.) Reliability guide. Mathematical foundations (WEMA, Warszawa, 1982)

9. R. Dobracki, Detailed geological map of Poland in a scale of 1:50 000, sheets Szczecin (228) with explanations (Warszawa, CAG Polish Geological Institute - National Research Institute, 1982, in Polish)

10. R. Hoc and A. Piotrowski, LXXX Scientific Meeting of the Polish Geological Society (Szczecin August, 11-14, 2010, in Polish)

11. A. Piotrowski, 1st Conference of MELA. Szczecin, Polish Geological Institute (2007)

12.

13. 